\section{Reevaluating Serologic Markers of Poor Prognostic Factors in Rheumatoid Arthritis}

\section{To the Editor:}

The article by Alemao, et $a l^{1}$ documented that acceleration of treatment regimens for 3458 biologic-naive patients with rheumatoid arthritis (RA) did not significantly differ based on the presence or absence of poor prognostic factors (PPF). These findings suggest that, for whatever reason, clinicians were unable to translate PPF into a more aggressive therapeutic approach. Further, this occurred even though patients with a high initial PPF fared worse over a 12-month period in achieving low disease activity and maintaining employment. The reasons for this lack of translation by clinicians of PPF to more aggressive treatment is uncertain. The more recent focus on "treat to target," evident in the 2015 American College of Rheumatology treatment recommendations, addresses high disease activity but does not directly address the role of other prognostic factors. This study suggests that treating to target and responding aggressively to PPF are linked. Three out of the 4 PPF observed in the study rely on previous or existing clinical features, including joint erosions by imaging, but some studies suggest that other comorbidities such as obesity and current smoking may also be relevant.

The final element included in PPF is seropositivity, and this is the area we feel has the greatest potential for improvement. Novel biomarkers including anticarbamylated protein (anti-CarP), antipeptidyl arginine deiminase type 4/3 antibodies, 14-3-3 eta, and antibodies to modified citrullinated vimentin promise a new era of serologic markers. When elevated, each of these markers has been associated with development of more aggressive and erosive disease. Considering the 2 serologic markers included in this study [anticitrullinated protein antibodies (ACPA) and rheumatoid factor (RF)], it may be time to reconsider serologic markers for their respective diagnostic versus prognostic value. Numerous studies have shown that patients with elevated levels of anticyclic citrullinated peptide antibodies (anti-CCP) are prone to more severe disease compared to those with normal levels; however, CCP has a known blind spot for so-called seronegative patients. The observation of severe erosive disease in the absence of anti-CCP is not uncommon and may unknowingly contribute to the lack of confidence in the marker's prognostic capabilities. Further, achieving low disease activity in some studies is independent of the presence or absence of RF or $\mathrm{ACPA}^{2}$.

The presence of RF as a PPF in RA seems overdue for debate. The pathogenic implications of RF remain elusive and it seems that RF should be replaced as a PPF with one of the more recent markers. By including markers that have little significant association with RA disease prognosis in the PPF, we may erode the confidence of patients and practitioners alike in the utility of acting upon PPF.

In 2017 Truchetet, et al found that anti-CarP was a significant marker for poorer clinical outcome and actually did correlate with use of more aggressive biologic therapy, even when it was not used as a $\mathrm{PPF}^{3}$. European cohorts have established that elevations in anti-CarP provide insight into "seronegative" RA and also a select subset of patients prone to more erosive disease regardless of ACPA status, as presented by Shi, et al (Figure 1) ${ }^{4}$.

Physicians today rarely rely on PPF, focusing more on treating the patient and their symptoms and certainly not their biomarkers. This is well aligned with the treat-to-target concept when the patient is fully on board with the concern for future disease progression. However, both the rheumatologist and patient are likely to be more therapeutically aligned in the presence of 1 or more serologic biomarkers associated with more aggressive disease.

The recent availability of biomarkers such as anti-CarP should be welcomed into the arsenal of the rheumatologist to help stay 1 step ahead of aggressive and erosive rheumatoid disease with equally aggressive early adoption of disease-modifying antirheumatic drug therapies.

ARTHUR WEINSTEIN, FACP, FRCP, MACR, MD, Georgetown University, Washington, DC; BRIAN McEVILLY, MBA; THIERRY DERVIEUX, DABCC, PhD, Exagen, Vista, California, USA. A. Weinstein is a consultant to Exagen, B. McEvilly and T. Dervieux are employees of Exagen. Address correspondence to B. McEvilly, 1261 Liberty Way, Vista, California 92081, USA.E-mail: Mcevilly7@yahoo.com

\section{REFERENCES}

1. Alemao E, Litman HJ, Connolly SE, Kelly S, Hua W, Rosenblatt L, et al. Do poor prognostic factors in rheumatoid arthritis affect treatment choices and outcomes? Analysis of a US Rheumatoid Arthritis Registry. J Rheumatol 2018;45:1353-60.

2. Baganz L, Richter A, Albrecht K, Schneider M, Burmester GR, Zink A, et al. Are prognostic factors adequately selected to guide treatment decisions in patients with rheumatoid arthritis? A collaborative analysis from three observational cohorts. Semin Arthritis Rheum 2018 Sept 17 (E-pub ahead of print).

3. Truchetet ME, Dublanc S, Barnetche T, Vittecoq O, Mariette X, Richez P, et al; Fédération Hospitalo-Universitaire ACRONIM. Association of the presence of anti-carbamylated protein antibodies in early arthritis with a poorer clinical and radiologic outcome: Data
A All RA patients

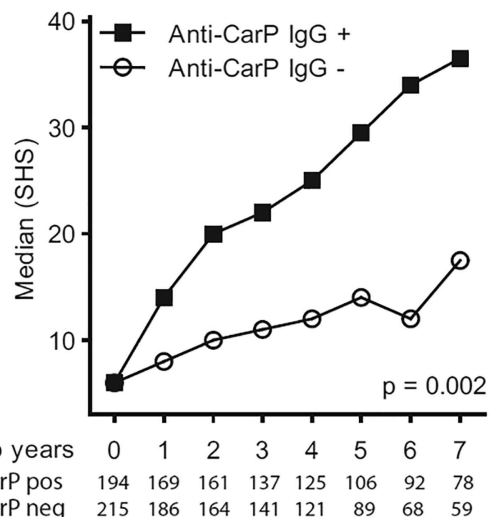

B

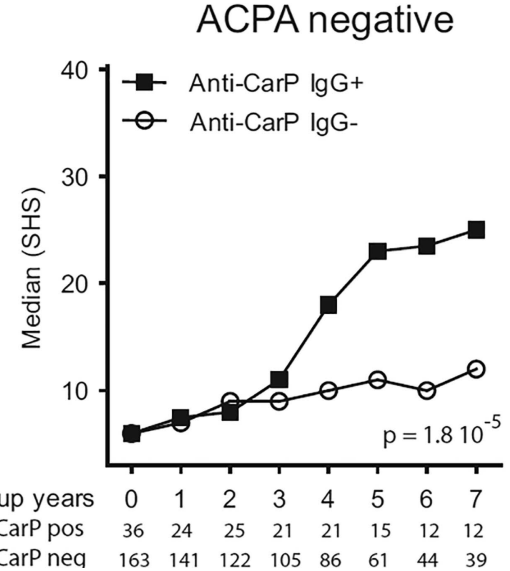

C ACPA positive

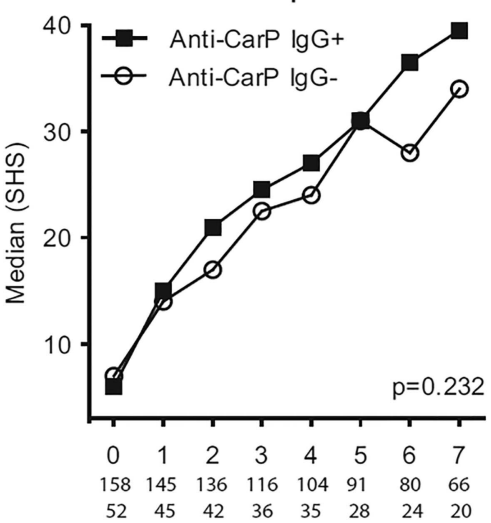

Figure 1. Joint damage by anti-CarP status. RA: rheumatoid arthritis; ACPA: anticitrullinated protein antibodies; IgG: immunoglobulin G; SHS: Sharp/van der Heijde score. Panel B reprinted with permission from Shi J, et al. Autoantibodies recognizing carbamylated proteins are present in sera of patients with rheumatoid arthritis and predict joint damage. Proc Natl Acad Sci U S A 2012;42:17372-7.

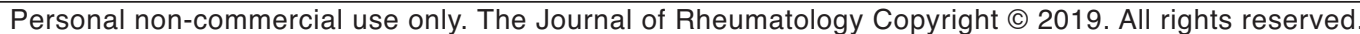


from the French ESPOIR cohort. Arthritis Rheumatol

2017;69:2292-302.

4. Shi J, Knevel R, Suwannalai P, van der Linden M, Janssen GM, van

Veelen $\mathrm{P}$, et al. Autoantibodies recognizing carbamylated proteins are present in sera of patients with rheumatoid arthritis and predict joint damage. Proc Natl Acad Sci U S A 2012;42:17372-7.

First Release April 15 2019; J Rheumatol 2019;46:6;

doi:10.3899/jrheum.181246 